\title{
Endogenous pleiotrophin and midkine regulate LPS-induced glial responses
}

Rosalía Fernández-Calle ${ }^{\dagger}$, Marta Vicente-Rodríguez ${ }^{\dagger}$, Esther Gramage ${ }^{\dagger}$, Carlos de la Torre-Ortiz ${ }^{\dagger}$, Carmen Pérez-García ${ }^{\dagger}$, María P. Ramos ${ }^{T}$, Gonzalo Herradón ${ }^{\dagger} * *$

† Pharmacology lab, Department of Pharmaceutical and Health Sciences. Facultad de Farmacia. Universidad CEU San Pablo, Madrid, Spain.

$\mathrm{T}$ Biochemistry and Molecular Biology lab, Department of Chemistry and Biochemistry. Facultad de Farmacia. Universidad CEU San Pablo, Madrid, Spain.

\author{
*Corresponding author: \\ Gonzalo Herradón, PhD \\ Lab. Pharmacology \\ Faculty of Pharmacy \\ Universidad CEU San Pablo \\ Urb. Montepríncipe \\ 28668 Boadilla del Monte, Madrid, Spain. \\ Tel: 34-91-3724700 (Ext. 4840) \\ Fax: 34-91-3510475 \\ e-mail: herradon@ceu.es
}

\section{Authors e-mail addresses:}

Rosalía Fernández-Calle: rosalia.fernandezcalle@ beca.ceu.es

Marta Vicente-Rodríguez: martavro@gmail.com

Esther Gramage: esther.gramagecaro@ ceu.es

Carlos de la Torre-Ortiz: c.torre3@usp.ceu.es

Carmen Pérez-García: capegar@ceu.es

María P. Ramos: pramos@ceu.es 


\begin{abstract}
Pleiotrophin (PTN) and Midkine (MK) are two growth factors that modulate neuroinflammation. PTN overexpression in the brain prevents LPS-induced astrocytosis in mice but potentiates microglial activation. The modest astrocytic response caused by a low dose of LPS $(0.5 \mathrm{mg} / \mathrm{kg})$ is blocked in the striatum of MK-/- mice whereas microglial response is unaffected. We have now tested the effects of an intermediate dose of LPS (7.5 mg/kg) in glial response in PTN-/- and MK-/- mice. We found that LPS-induced astrocytosis is prevented in prefrontal cortex and striatum of both PTN-/- and MK-/- mice. Some of the morphological changes of microglia induced by LPS tended to increase in both genotypes, particularly in PTN-/- mice. Since we previously showed that PTN potentiates LPS-induced activation of BV2 microglial cells, we tested the activation of FYN kinase, a substrate of the PTN receptor RPTP $\beta / \zeta$, and the subsequent ERK1/2 phosphorylation on LPS and PTN-treated BV2 cells. LPS effects on BV2 cells were not affected by the addition of PTN, suggesting that PTN does not recruit the FYN-MAP kinase signaling pathway in order to modulate LPS effects on microglial cells. Taking together, evidences demonstrate that regulation of astroglial responses to LPS administration are highly dependent on the levels of expression of PTN and MK. Further studies are needed to clarify the possible roles of endogenous expression of PTN and MK in LPS-induced microglial responses.
\end{abstract}

Keywords: Neuroinflammation, microglia, astrogliosis, FYN kinase, pleiotrophin, midkine. 


\section{INTRODUCTION}

Activation of the innate immunity in the Central Nervous System (CNS) is an important step in the healing process of the damaged brain tissue. When prolonged, however, neuroinflammation can become deleterious [1]. Activation of microglia and astroglia are key events in neuroinflammatory processes and contribute to the neuronal alterations characteristic of pathologies with overt neuroinflammation including neurodegenerative diseases [2], multiple sclerosis [3,4] and drug addiction [5].

The contribution of Toll-like receptors (TLRs) to neuroinflammatory processes has been shown [6]. However, the genetic background that modulates neuroinflammation is largely elusive. Recently, we identified pleiotrophin (PTN) and Midkine (MK) as two novel growth factors that modulate neuroinflammation in different contexts [7-9]. Pleiotrophin and MK are the only cytokines that constitute the PTN/MK developmental gene family $[10,11]$ and overlap many functions including survival and differentiation of neurons [12]. Both PTN and MK bind to receptor protein tyrosine phosphatase (RPTP) $\beta / \zeta$ (a.k.a. PTPRZ1, (R)PTP $\beta$ or PTP $\zeta$ ) and inhibit its phosphatase activity $[13,14]$, leading to increased phosphorylation of substrates of RPTP $\beta / \zeta$ such as FYN kinase [15]. Pleiotrophin and MK are upregulated in different pathologies of the CNS characterized by neuroinflammation including neurodegenerative diseases, addictive disorders, ischemia and neuropathic pain [5], which led us to hypothesize a modulatory role of these cytokines in neuroinflammatory processes. Previously, we observed that highly upregulated PTN brain levels enhance amphetamine-induced astrocytosis [7] but prevents LPS-induced astrocytic response [9]. Importantly, a 4-fold upregulation of PTN levels in PFC potentiates LPS-induced microglial activation and release of inflammatory cytokines [9]. On the other hand, the modest astrocytic response elicited by a very low dose of LPS 
$(0.5 \mathrm{mg} / \mathrm{kg})$ seems to be blocked in the striatum of MK-/- mice whereas no significant changes were observed in microglial response [8]. The data suggest a complex regulatory role of PTN and MK in important processes of neuroinflammation such as microglial and astrocytic responses, depending on their own levels of expression and the nature and intensity of the inflammatory stimulus. In order to assess the importance of normal endogenous expression of these cytokines in response to inflammatory stimuli, we aimed to test the effects of an intermediate dose of LPS $(7.5 \mathrm{mg} / \mathrm{kg})$ in glial response in different brain areas of PTN-/- and MK-/- mice. In addition, since FYN kinase has been recently shown as an upstream regulator of proinflammatory signaling [16] and is a substrate of $\mathrm{RPTP} \beta / \zeta$ [15], we have explored the possibility that PTN regulates LPS effects in vitro through its ability to modulate FYN kinase signaling. 


\section{MATERIAL AND METHODS}

\subsection{Animals}

PTN-/- and MK-/- mice were kindly provided by Dr. Thomas F. Deuel (The Scripps Research Institute, La Jolla, CA). MK-/- and PTN-/- mice on a C57BL/6J background were generated by methods previously described $[17,18]$. We used male MK-/-, PTN-/- and WT animals of 9-10 weeks (20-25 g). Mice were housed under controlled environmental conditions $\left(22 \pm 1^{\circ} \mathrm{C}\right.$ and a 12 -h light/12-h dark cycle) with free access to food and water. Mice were maintained in accordance with both the ARRIVE guidelines and the European Union Laboratory Animal Care Rules (Directive 2010/63/EU for animal experiments) and the protocols were approved by the Animal Research Committee of USP-CEU.

\subsection{Immunohistochemistry}

Mice from all experimental groups ( $n=4-5 /$ group) received a single i.p. injection of LPS (Sigma, Madrid, Spain) $(7.5 \mathrm{mg} / \mathrm{kg}$ ) or saline (control, $10 \mathrm{ml} / \mathrm{kg}$ ) and were sacrificed $16 \mathrm{~h}$ after LPS administration. This protocol was previously used by our group in mice with transgenic overexpression of PTN in the brain [9].

Animals were sacrificed by transcardial perfusion with $4 \%$ paraformaldehyde. Brains were removed and $30 \mu \mathrm{m}$ PFC and striatal free-floating sections were processed as previously described [7-9]. Immunohistochemistry studies were performed in one slice per $180 \mu \mathrm{m}$ (PFC from bregma $-3.08 \mathrm{~mm}$ to $-2.46 \mathrm{~mm}$; Striatum from bregma $1.54 \mathrm{~mm}$ to $-0.10 \mathrm{~mm}$ ). Sections were incubated overnight at $4^{\circ} \mathrm{C}$ with anti-glial fibrillary acidic protein (GFAP; Millipore, Madrid, Spain; 1:1000) and anti-ionized calcium-binding adaptor molecule 1 (Iba1, Wako, Osaka, Japan; 1:1000) antibodies, following by 30 minutes incubation with the Alexa-Fluor-555 and Alexa-Fluor-488 corresponding 
secondary antibody (Invitrogen, Waltham, MA USA; 1:500). Photomicrographs were captured with a digital camera coupled to an optical microscope (DM5500B, Leica, Solms, Germany). Analysis was performed using ImageJ (NIH), in the three most central slices of each area. Iba1+ cells and GFAP+ astrocytes were counted in whole sections of PFC and in $1100 \mu \mathrm{m} \times 1400 \mu \mathrm{m}$ standardized areas in the striatum [9]. Analysis of changes in microglia morphology was performed as recently described [9]. Briefly, total marked area was calculated as overall image fluorescence, subtracting the mean background fluorescence. The soma size (cell area) is expressed in square micrometers. The perimeter was calculated based on the outline length of a given object, is expressed in microns and is expected to be higher in activated cells. Circularity was calculated by the following formula: $4 \pi \times($ area/perimeter2). This parameter varies from 0 (linear polygon) to 1 (perfect circular object). Mean single cell values for each parameter were used for statistics.

\subsection{BV2 murine microglial cell cultures}

BV2 murine microglial cells were a generous gift from Professor Antonio Cuadrado (Instituto de Investigaciones Biomédicas "Alberto Sols" (IIBM), Madrid, Spain). Cells were routinely maintained in RPMI-1640 medium with fetal bovine serum (10\%), penicillin $(100 \mathrm{U} / \mathrm{ml})$, streptomycin $(100 \mu \mathrm{g} / \mathrm{ml})$ and L-glutamine $(4 \mathrm{mM})$ at $37^{\circ} \mathrm{C}$ in 5\% CO2 humidified air following conditions used by others $[19,20]$.

To test the effects of PTN (Sigma, Spain) on LPS-stimulated BV2 cell cultures, cells were incubated for 30 minutes with LPS $(1.0 \mu \mathrm{g} / \mathrm{ml})$ and/or PTN $(0.5 \mu \mathrm{g} / \mathrm{ml})$. Total protein from non-stimulated, PTN-, and LPS-stimulated BV2 cell lysates (Six 60-mm plates/each) was quantified by the BCA protein assay (Pierce, Rockford, IL). Equilibrated protein samples were mixed with loading buffer (60mM Tris pH 6.8, 10\% glycerol, 5\% 
SDS, $0.65 \% \beta$-mercaptoethanol, and $0.01 \%$ bromophenol blue), boiled for 5 minutes, and loaded onto $10 \%$ polyacrylamide gels (Invitrogen, Carlsbad, CA) with SDS (SDSPAGE). The gels were transferred to nitrocellulose membranes, blocked with 50mM Tris, $150 \mathrm{mM} \mathrm{NaCl}, 0.1 \%$ Tween-20 (TBS-T), and 5\% non-fat milk for 1 hour. We determined the phosphorylation status of the Y416 residue in FYN kinase activation loop domain by using the phospho Y416 Src family kinase (p-Y416SFK) antibody (Cell signaling, Danvers, MA), which has been used extensively to demonstrate FYN kinase activation $[16,21,22]$. In addition, membranes were probed with anti-phospho-ERK1/2 (1:1000) (Cell signaling, Danvers, MA) antibodies and reprobed with anti-FYN and anti-ERK1/2 (1:1000) (Cell signaling, Danvers, MA) antibodies to confirm the identity of the protein. Membranes were incubated for one hour with appropriate secondary antibodies (Santa Cruz biotechnology, Santa Cruz, CA) conjugated with horseradish peroxidase diluted 1:5000 in TBS-T with 5\% non-fat milk for $30 \mathrm{~min}$. The immunoreactive proteins were visualized using the ECL Enhanced method according to the manufacturer's instructions (Amersham, San Francisco, CA).

\subsection{Statistics}

Data are presented as mean \pm standard error of the mean (S.E.M.). Data obtained from immunohistochemistry studies were analyzed using two-way ANOVA considering genotype and treatment as variants. Relevant differences were analyzed by post-hoc comparisons with Bonferroni's post-hoc tests. Data obtained from BV2 cells were analyzed using one-way ANOVA followed by post-hoc comparisons with Tukey's posthoc tests. $\mathrm{P}<0.05$ was considered as statistically significant. All statistical analyses were performed using Graph-Pad Prism program (San Diego, CA, USA). 


\section{RESULTS}

\subsection{Differential regulation of LPS-induced astrocytic and microglial responses in the PFC and striatum of PTN-/- and MK-/- mice.}

First, we found that LPS $(7.5 \mathrm{mg} / \mathrm{kg})$ increased the number of GFAP+ astrocytes in the PFC (Fig. 1) and striatum (Fig. 2) of WT mice. The upregulation of GFAP levels in astrocytes was apparent since most of these cells were heavily stained, suggesting astroglial activation. In contrast, these effects on GFAP+ immunoreactivity induced by LPS were blocked in PTN-/- and MK-/- mice independently of the brain area considered (Figs. 1 and 2). The data clearly show that normal levels of endogenous PTN and MK are required for LPS-induced astrocytic response.

PFC and striatal sections were then immunostained with anti-Iba1 microglial antibody. We observed resting morphology of microglia in the PFC of saline-treated animals independently of the genotype (Fig. 3). Immunohistochemistry for Iba1 did not reflect significant changes in the number of Iba1+ cells in the PFC after LPS treatment (Fig. 3B). However, we observed signs of enhanced hypertrophism in LPS-treated mice, particularly in the case of mice lacking endogenous PTN (Fig. 3). We did not detect significant changes in the total marked area (Fig. 3C), probably because the number of Iba1+ cells tended to be lower in PTN-/- mice. The Iba1+ cell area, a parameter expected to be higher in activated and hypertrophic cells, was significantly increased by LPS in all genotypes (Fig. 3D). The magnitude of the increase in the perimeter caused by LPS was similar in PFC of all genotypes although only reached significance in the case of WT mice (Fig. 3E). The reduction caused by LPS in the circularity index, a parameter expected to decrease in activated cells, was significantly more pronounced in PTN-/- and MK-/- mice (Fig. 3F). Interestingly, these differences between genotypes in the PFC were nearly lost in the striatum (Fig. 4), a brain area where we only detected that LPS-induced 
increase of Iba1+ cell area was significantly enhanced in PTN-/- mice (Fig. 4D). Overall, the data show that some of the LPS-induced microglial morphological changes are differentially regulated in PTN-/- and MK-/- mice.

\subsection{FYN signaling is not involved in the regulation of LPS effects by PTN in BV2 microglial cells.}

We recently showed that LPS $(1.0 \mu \mathrm{g} / \mathrm{ml})$-induced increase of nitric oxide production in murine BV2 microglial cells is significantly enhanced by PTN $(0.5 \mu \mathrm{g} / \mathrm{ml})$, suggesting that PTN potentiates microglial activation in vitro [9]. In the present study, we determined the phosphorylation status of the Y416 residue in FYN kinase activation loop domain, which was found very low independently of the treatment (Fig. 5A). PTN alone tended to increase p-Y416 FYN levels; however, PTN did not exert significant effects in LPS-stimulated BV2 cells (Fig. 5A). In addition, we studied LPS-induced MAP kinase activation [16] by assessing the phosphorylation levels of ERK1/2. As expected, we found that LPS induced robust increases of the phosphorylation levels of ERK1/2 (Fig. 5B). However, these LPS effects were not affected by the addition of PTN to LPS-treated BV2 cells. Overall, the data suggest that PTN does not recruit the FYN-ERK1/2 signaling pathway in order to regulate LPS effects in BV2 cells [9]. 


\section{DISCUSSION}

We previously showed that astrocytosis induced by a very low dose of LPS $(0.5$ $\mathrm{mg} / \mathrm{kg}$ ) is prevented in MK-/- mice [8]. In the present study, astrocytosis induced by a single administration of an intermediate dose of LPS $(7.5 \mathrm{mg} / \mathrm{kg})$ is blocked in the brain of PTN-/- and MK-/- mice. The data demonstrate that normal endogenous levels of PTN and MK are required for LPS-induced astrocytic response independently of the intensity of the inflammatory stimulus. However, we previously found that transgenic PTN overexpression in the PFC of PTN-Tg mice attenuates LPS-induced astrocytic response [9]. It has to be noted that, in contrast to the wide distribution of PTN in the CNS during early development, this cytokine is only expressed at low levels in a few cell types in the adult cortex, hippocampus, cerebellum, olfactory bulb and striatum [5,23]. In contrast, PTN is found highly upregulated in a wide variety of CNS disorders characterized by neuroinflammation including neuropathic pain [24,25], Alzheimer's disease [26], Parkinson's disease [27] and brain tumors [28]. Thus, we believe that the data presented here in PTN-/- mice compared to normal wild type mice, add to the knowledge of the physiological functions of normal endogenous PTN levels and their contribution to the response to acute neuroinflammatory stimuli. In contrast, studies in transgenic mice with highly significant overexpression of PTN in the brain may be more relevant to dissect the contribution of high levels of PTN in neuroinflammatory pathological conditions [7,9].

Lack of endogenous expression of these cytokines, particularly PTN, tend to modulate microglia morphological changes in the PFC of LPS-treated knockout mice compared to WT mice. In the PFC of WT mice, LPS increased the perimeter and area of Iba1+ cells, suggesting the presence of hypertrophic microglia. On the other hand, LPS induced a significant decrease in the circularity index in PTN-/- and MK-/- mice compared to WT mice. In the striatum, LPS tended to increase the area and perimeter of 
microglial cells in all genotypes although this effect was only found to be significant in the area of microglial cells in PTN-/-. Little was known about the role of endogenous PTN and MK in the microglial response to inflammatory insults. This is the first study showing that normal endogenous expression of PTN and MK in vivo modulates LPSinduced microglial morphological changes. It is important to note that we did not find differences between genotypes in saline-treated animals, suggesting that PTN and MK do not regulate innate immunity in naïve animals. Taking into account that PTN and MK are expressed at low levels in the healthy adult brain [5], the present study suggests that lack of endogenous expression of these cytokines impact the microglial changes in response to the endotoxin. Taking together all the microglial morphological parameters analyzed, it is tempting to speculate that absence of these cytokines, particularly PTN, tends to predispose to an enhanced microglial activation. However, it is clear that further studies are needed to clarify the roles of these endogenous cytokines in LPS-induced microglial activation (e.g. cell-specific knockdown).

We previously showed that exogenous PTN potentiates LPS-induced activation of BV2 microglial cells [9]. Previous evidence showed that FYN kinase contributes to MAP kinase phosphorylation during microglial activation [16]. Since FYN is a substrate of the PTN receptor RPTP $\beta / \zeta$ [15], we tested the effects of PTN and LPS on FYN signaling in BV2 microglial cells. Our results clearly show that treatment of BV2 cells with PTN and/or LPS does not cause relevant changes in the phosphorylation of Y416 in FYN. On the other hand, LPS-induced increases of phosphorylation of ERK1/2 were not modulated by the addition of PTN to LPS-stimulated BV2 cells. Taking together, our data suggest that PTN actions in primed microglia are independent of the FYN-MAP kinase signaling pathway. 


\section{CONCLUSION}

Our findings show for the first time that endogenous PTN and MK modulate the astrocytic response induced by LPS. The data suggest that PTN and/or MK may be involved in the modulation of LPS-induced microglial activation but further studies are needed to delineate their importance.

Funding: This work has been supported by grants SAF2014-56671-R from Ministerio de Economía y Competitividad of Spain, PNSD001I2015 from National Plan on Drug abuse, Ministerio de Sanidad, Servicios Sociales e Igualdad of Spain. M V-R and R F-C were supported by fellowships from Fundación Universitaria San Pablo CEU. 


\section{LIST OF ABBREVIATIONS}

ANOVA: analysis of variance

CNS: central nervous system

GFAP: glial fibrillary acidic protein

Iba1: ionized calcium-binding adaptor molecule 1

IL-1 $\beta$ : interleukin $1 \beta$

IL-6: interleukin 6

iNOS: inducible nitric oxide synthase

LPS: lipopolysaccharide

MAP kinase: mitogen-activated protein kinase

MK-/-: MK genetically deficient mice

MK: Midkine

NFкB: nuclear factor- $\kappa \mathrm{B}$

PFC: prefrontal cortex

PTN-/-: PTN genetically deficient mice

PTN: Pleiotrophin

PTN-Tg: mice with transgenic PTN overexpression in the brain

RPMI-1640: Roswell Park Memorial Institute 1640 medium

RPTP $\beta / \zeta$ (PTPRZ1): receptor protein tyrosine phosphatase $\beta / \zeta$

S.E.M.: standard error of the mean

TLRs: Toll-like receptors

TNF- $\alpha$ : tumor necrosis factor- $\alpha$

WT: wild type 


\section{REFERENCES}

1. Faden AI, Wu J, Stoica BA, Loane DJ (2016) Progressive inflammation-mediated neurodegeneration after traumatic brain or spinal cord injury. Br J Pharmacol 173: 681-691.

2. McManus RM, Heneka MT (2017) Role of neuroinflammation in neurodegeneration: new insights. Alzheimers Res Ther 9: 14.

3. Mori F, Nistico R, Mandolesi G, Piccinin S, Mango D, et al. (2014) Interleukin-1beta promotes long-term potentiation in patients with multiple sclerosis. Neuromolecular Med 16: 38-51.

4. Mandolesi G, De Vito F, Musella A, Gentile A, Bullitta S, et al. (2017) miR-142-3p Is a Key Regulator of IL-1beta-Dependent Synaptopathy in Neuroinflammation. J Neurosci 37: 546-561.

5. Herradon G, Perez-Garcia C (2014) Targeting midkine and pleiotrophin signalling pathways in addiction and neurodegenerative disorders: recent progress and perspectives. Br J Pharmacol 171: 837-848.

6. Akira S, Takeda K (2004) Functions of toll-like receptors: lessons from KO mice. C R Biol 327: 581-589.

7. Vicente-Rodriguez M, Rojo Gonzalez L, Gramage E, Fernandez-Calle R, Chen Y, et al. (2016) Pleiotrophin overexpression regulates amphetamine-induced reward and striatal dopaminergic denervation without changing the expression of dopamine D1 and D2 receptors: Implications for neuroinflammation. Eur Neuropsychopharmacol 26:1794-1805

8. Vicente-Rodriguez M, Fernandez-Calle R, Gramage E, Perez-Garcia C, Ramos MP, et al. (2016) Midkine Is a Novel Regulator of Amphetamine-Induced Striatal Gliosis and Cognitive Impairment: Evidence for a Stimulus-Dependent Regulation of Neuroinflammation by Midkine. Mediators Inflamm 2016: 9894504.

9. Fernandez-Calle R, Vicente-Rodriguez M, Gramage E, Pita J, Perez-Garcia C, et al. (2017) Pleiotrophin regulates microglia-mediated neuroinflammation. J Neuroinflammation 14: 46.

10. Kadomatsu K, Tomomura M, Muramatsu T (1988) cDNA cloning and sequencing of a new gene intensely expressed in early differentiation stages of embryonal carcinoma cells and in mid-gestation period of mouse embryogenesis. Biochem Biophys Res Commun 151: 1312-1318.

11. Milner PG, Li YS, Hoffman RM, Kodner CM, Siegel NR, et al. (1989) A novel 17 $\mathrm{kD}$ heparin-binding growth factor (HBGF-8) in bovine uterus: purification and Nterminal amino acid sequence. Biochem Biophys Res Commun 165: 1096-1103.

12. Gramage E, Herradon G (2011) Connecting Parkinson's disease and drug addiction: common players reveal unexpected disease connections and novel therapeutic approaches. Curr Pharm Des 17: 449-461.

13. Maeda N, Ichihara-Tanaka K, Kimura T, Kadomatsu K, Muramatsu T, et al. (1999) A receptor-like protein-tyrosine phosphatase PTPzeta/RPTPbeta binds a heparinbinding growth factor midkine. Involvement of arginine 78 of midkine in the high affinity binding to PTPzeta. J Biol Chem 274: 12474-12479.

14. Meng K, Rodriguez-Pena A, Dimitrov T, Chen W, Yamin M, et al. (2000) Pleiotrophin signals increased tyrosine phosphorylation of beta beta-catenin through inactivation of the intrinsic catalytic activity of the receptor-type protein tyrosine phosphatase beta/zeta. Proc Natl Acad Sci U S A 97: 2603-2608. 
15. Pariser H, Ezquerra L, Herradon G, Perez-Pinera P, Deuel TF (2005) FYN is a downstream target of the pleiotrophin/receptor protein tyrosine phosphatase beta/zeta-signaling pathway: regulation of tyrosine phosphorylation of FYN by pleiotrophin. Biochem Biophys Res Commun 332: 664-669.

16. Panicker N, Saminathan H, Jin H, Neal M, Harischandra DS, et al. (2015) FYN Kinase Regulates Microglial Neuroinflammatory Responses in Cell Culture and Animal Models of Parkinson's Disease. J Neurosci 35: 10058-10077.

17. Amet LE, Lauri SE, Hienola A, Croll SD, Lu Y, et al. (2001) Enhanced hippocampal long-term potentiation in mice lacking heparin-binding growth-associated molecule. Mol Cell Neurosci 17: 1014-1024.

18. Nakamura E, Kadomatsu K, Yuasa S, Muramatsu H, Mamiya T, et al. (1998) Disruption of the midkine gene (Mdk) resulted in altered expression of a calcium binding protein in the hippocampus of infant mice and their abnormal behaviour. Genes Cells 3: 811-822.

19. Lastres-Becker I, Garcia-Yague AJ, Scannevin RH, Casarejos MJ, Kugler S, et al. (2016) Repurposing the NRF2 Activator Dimethyl Fumarate as Therapy Against Synucleinopathy in Parkinson's Disease. Antioxid Redox Signal 25: 61-77.

20. Luo XL, Liu SY, Wang LJ, Zhang QY, Xu P, et al. (2016) A tetramethoxychalcone from Chloranthus henryi suppresses lipopolysaccharide-induced inflammatory responses in BV2 microglia. Eur J Pharmacol 774: 135-143.

21. Wake H, Lee PR, Fields RD (2011) Control of local protein synthesis and initial events in myelination by action potentials. Science 333: 1647-1651.

22. Larson M, Sherman MA, Amar F, Nuvolone M, Schneider JA, et al. (2012) The complex PrP(c)-FYN couples human oligomeric Abeta with pathological tau changes in Alzheimer's disease. J Neurosci 32: 16857-16871a.

23. González-Castillo C, Ortuño-Sahagún D, Guzmán-Brambila C, Pallàs M, RojasMayorquín AE. (2015) Pleiotrophin as a central nervous system neuromodulator, evidences from the hippocampus. Front Cell Neurosci 8:443.

24. Ezquerra L, Alguacil LF, Nguyen T, Deuel TF, Silos-Santiago I, Herradon G. (2008) Different pattern of pleiotrophin and midkine expression in neuropathic pain: correlation between changes in pleiotrophin gene expression and rat strain differences in neuropathic pain. Growth Factors 26(1): 44-48.

25. Martin YB, Herradon G \& Ezquerra L (2011). Uncovering new pharmacological targets to treat neuropathic pain by understanding how the organism reacts to nerve injury. Curr Pharm Des 17: 434-448.

26. Wisniewski T, Lalowski M, Baumann M, Rauvala H, Raulo E, Nolo R et al. (1996). HB-GAM is a cytokine present in Alzheimer's and Down's syndrome lesions. Neuroreport 7: 667-671.

27. Marchionini DM, Lehrmann E, Chu Y, He B, Sortwell CE, Becker KG, et al. (2007). Role of heparin binding growth factors in nigrostriatal dopamine system development and Parkinson's disease. Brain Res 1147: 77-88.

28. Ma J, Lang B, Wang X, Wang L, Dong Y, Hu H. (2014) Co-expression of midkine and pleiotrophin predicts poor survival in human glioma. J Clin Neurosci 21(11):1885-90. 


\section{FIGURE LEGENDS}

Fig. 1. LPS effects on astrocytosis in the PFC of WT, MK-/- and PTN-/- mice. Photomicrographs are from GFAP-immunostained PFC sections of saline (Sal)- or LPStreated animals ( $n=4-5 /$ group). The graph represents quantification of data obtained from the counts of GFAP-positive cells in PFC whole sections. \#\# $\mathrm{P}<0.01$ vs. WT with the same treatment. Scale bar $=500 \mu \mathrm{m}$ (high magnification: scale bar $=50 \mu \mathrm{m}$ ).

Fig. 2. LPS effects on astrocytosis in the striatum of WT, MK-/- and PTN-/- mice. Photomicrographs are from GFAP-immunostained striatal sections of saline (Sal)- or LPS-treated animals ( $n=4-5 /$ group). The graph represents quantification of data obtained from the counts of GFAP-positive cells in standardized striatal sections. $* \mathrm{P}<0.05$ vs. Sal within the same genotype. \# $\mathrm{P}<0.05$ vs. WT. \#\# $\mathrm{P}<0.01$ vs. WT with the same treatment. Scale bar $=200 \mu \mathrm{m}$ (high magnification: scale bar $=50 \mu \mathrm{m})$.

Fig. 3. Effects of LPS on microglia activation in the PFC of WT, MK-/- and PTN-/mice. Photomicrographs are from Iba-1-immunostained PFC sections of saline (Sal)- or LPS-treated animals ( $n=4$-5/group) (A). Graphs represent quantification of data (mean \pm S.E.M) obtained from the counts of Iba-1-positive cells (B), total marked area (C), cell area (D), soma perimeter $(\mathrm{E})$ and circularity index $(\mathrm{F})$ in $\mathrm{PFC}$ whole sections. $* \mathrm{P}<0.05$, $* * \mathrm{P}<0.01, * * * \mathrm{P}<0.001$ vs. Sal within the same genotype. Scale bar $=100 \mu \mathrm{m}$ (high magnification: scale bar $=50 \mu \mathrm{m})$.

Fig. 4. Effects of LPS on microglia activation in the striatum of WT, MK-/- and PTN/- mice. Photomicrographs are from Iba-1-immunostained striatal sections of saline (Sal)or LPS-treated animals ( $n=4-5 /$ group) (A). Graphs represent quantification of data (mean \pm S.E.M) obtained from the counts of Iba-1-positive cells (B), total marked area (C), cell area (D), soma perimeter (E) and circularity index (F) in standardized striatal sections. * 
$\mathrm{P}<0.05$ vs. Sal within the same genotype. Scale bar $=100 \mu \mathrm{m}$ (high magnification: scale $\operatorname{bar}=50 \mu \mathrm{m})$.

Fig. 5. Effects of LPS and PTN on FYN and MAP kinase phosphorylation in BV2 microglial cells. (A), immunoblots showing p-Y416SFK (FYN) levels in BV2 cell lysates. Total FYN western blots are shown below the phosphorylated protein blot for comparison. (B), immunoblots showing the p44/42 (ERK) phosphorylation. Total ERK1/2 western blots are shown below the phosphorylated protein blot for comparison. 